\title{
Effect of maternal gestational weight gain on offspring DNA methylation: a follow-up to the ALSPAC cohort study
}

\author{
Jon Bohlin ${ }^{1 *+}$, Bettina K Andreassen ${ }^{1,2+}$, Bonnie R Joubert ${ }^{3}$, Maria C Magnus ${ }^{1}$, Michael C Wu ${ }^{4}$, Christine L Parr ${ }^{1}$, \\ Siri E Håberg ${ }^{1}$, Per Magnus ${ }^{1}$, Sarah E Reese ${ }^{3}$, Camilla Stoltenberg ${ }^{1}$, Stephanie J London ${ }^{3}$ and Wenche Nystad ${ }^{1}$
}

\begin{abstract}
Background: Several epidemiologic studies indicate that maternal gestational weight gain (GWG) influences health outcomes in offspring. Any underlying mechanisms have, however, not been established. A recent study of 88 children based on the Avon Longitudinal Study of Parents and Children (ALSPAC) cohort examined the methylation levels at 1,505 Cytosine-Guanine methylation (CpG) loci and found several to be significantly associated with maternal weight gain between weeks 0 and 18 of gestation. Since these results could not be replicated we wanted to examine associations between 0 and 18 week GWG and genome-wide methylation levels using the Infinium HumanMethylation450 BeadChip (450K) platform on a larger sample size, i.e. 729 newborns sampled from the Norwegian Mother and Child Cohort Study (MoBa).
\end{abstract}

Results: We found no CpG loci associated with 0-18 week GWG after adjusting for the set of covariates used in the ALSPAC study (i.e. child's sex and maternal age) and for multiple testing $(q>0.9$, both 1,505 and 473,731 tests). Hence, none of the CpG loci linked with the genes found significantly associated with 0-18 week GWG in the ALSPAC study were significant in our study.

Conclusions: The inconsistency in the results with the ALSPAC study with regards to the 0-18 week GWG model may arise for several reasons: sampling from different populations, dissimilar methylome coverage, sample size and/or false positive findings.

\section{Background}

Recent genome-wide DNA methylation mapping technologies have resulted in an increasing number of studies examining epigenetic effects on offspring from various maternal exposures during pregnancy. For instance, results from the Norwegian Mother and Child Cohort Study $(\mathrm{MoBa})$ indicate that maternal smoking during pregnancy influences methylation patterns in the offspring [1]. Furthermore, findings from $\mathrm{MoBa}$ show an association between patterns of methylation in the offspring and birth weight [2]. Although maternal

\footnotetext{
*Correspondence: jon.bohlin@fhi.no

${ }^{\dagger} J$ on Bohlin and Bettina K Andreassen contributed equally to this work ${ }^{1}$ Division of Epidemiology, Norwegian Institute of Public Health, Marcus Thranes gate 6, P.O. Box 4404, 0403 Oslo, Norway

Full list of author information is available at the end of the article
}

gestational weight gain (GWG) has been associated with offspring's health and development during childhood [3, 4] limited knowledge is currently available regarding epigenetic effects on offspring from maternal GWG.

Morales et al. conducted an epigenetic inquiry of putative effects from maternal pre-pregnancy BMI and GWG in cord blood DNA of 88 newborns by using data from the Avon Longitudinal Study of Parents and Children (ALSPAC) cohort [5]. They examined 807 candidate genes (1,505 CpG probes) for cancer using the Illumina GoldenGate Genotyping Assay. Several time intervals for GWG in pregnancy were tested, and a significant association between weeks $0-18 \mathrm{GWG}$, and a set of CpG probes linked to the genes: MMP7, KCNK4, TRPM5 and NFKB1 were reported. Morales et al. did not succeed in replicating their results in 170 non-overlapping ALSPAC subjects and encouraged more research based on larger 
studies and genome-wide DNA methylation data [5]. Hence, the aim of the present work was to explore the findings from Morales et al. using genome-wide DNA methylation data as well as determine the presence of any novel associations between newborn methylome CpG loci and maternal 0-18 week GWG. DNA methylation was measured in cord blood using the Infinium HumanMethylation450 BeadChip (450K), from 729 newborns participating in the MoBa cohort.

\section{Findings}

\section{GWG models as described in the ALSPAC study}

Morales et al. found a statistically significant association with probes linked to the genes $M M P 7, K C N K 4$, TRPM5 and NFKB1 for the model describing 0-18 week GWG. They tested the following covariates: child's sex, ethnic background, mode of delivery, maternal age, parity, maternal smoking in pregnancy and occupation, but found only maternal age and offspring sex to be of importance with respect to the results. We ran a similar genome-wide model for the 473,731 probes on the Infinium HumanMethylation450 BeadChip (sex chromosomes excluded) that overlapped with the genes represented on the Illumina GoldenGate Genotyping Assay examined by Morales et al. [5]. We adjusted for child's sex and maternal age in accordance with the model presented by Morales et al. A full model containing the covariates child's sex, mode of delivery, maternal age, parity, maternal education and maternal daily smoking during pregnancy (ethnic background was irrelevant due to a highly homogeneous population) was also fitted but since there were no differences with respect to significant findings (see Additional file 1; Table 1) we present the simpler model, in accordance with the study by Morales et al. Hence, we regressed CpG loci against 0-18 week GWG adjusted for child's sex and maternal age. The genomic inflation factor (GIF) $\lambda$ [6] was used as an indicator of hidden confounders and for model assessment $[7,8]$.

\section{Our results compared to those of the ALSPAC study}

From Fig. 1 it can be seen that the GIF for our $0-18$ week GWG model was low: $\lambda=1.037$, but revealed no significant methylation differences after q value correction ( $q>0.9,473,731$ tests, see Table 2; Additional file 2 for more details) [9]. Morales et al. did however run their models on the Illumina GoldenGate Genotyping Assay which maps 1,505 CpG sites, implying that the number of statistical tests corrected for is considerably smaller than the 473,731 tests resulting from our models. Nevertheless, it can be seen from the $\mathrm{p}$ values in Table 2 that correcting for multiple testing in accordance with the Illumina GoldenGate Genotyping Assay (1,505 tests) has no consequence for the CpG's associated with
Table 1 Summary statistics for the covariates used in the 0-18 weeks GWG regression models, $n=729$

\begin{tabular}{ll}
\hline Covariate & Estimate \\
\hline Child's sex, male & $48.8 \%$ \\
Mean age of mother at birth (years) & 30.1 (95\% Cl 29.8-30.4) \\
Mean 0-18 weeks GWG (kg/week) & $0.16(95 \% \mathrm{Cl} 0.14-0.17)$ \\
Not completed high-school & $7.1 \%$ \\
Completed high school & $33.6 \%$ \\
College/university & $41.8 \%$ \\
College/university more than 4 years & $17.1 \%$ \\
Maternal daily smoking, yes (8 missing) & $12.5 \%$ \\
Caesarian section, yes & $6 \%$ \\
1st & $40.7 \%$ \\
2nd & $39.5 \%$ \\
3rd & $14.1 \%$ \\
4th or more & $5.6 \%$ \\
\hline
\end{tabular}

the $0-18$ week GWG outcome in our dataset $(q>0.9)$. Hence, our results indicate that none of the probes were statistically significant for the $0-18$ week GWG model.

There were substantially more $\mathrm{CpG}$ loci associated with each gene: MMP7, KCNK4, TRPM5 and NFKB1 on the Illumina HumanMethylation450 BeadChip platform than on the Illumina GoldenGate Assay used by Morales et al. [5], but 5 were missing (Table 2). The five CpGs missing in our study were typically replaced by several $\mathrm{CpG}$ loci nearby (see Table 2; Additional file 2). The HumanMethylation 450 platform contained a total of $98 \mathrm{CpGs}$, most of which were located in the promoter region of the genes: MMP7, KCNK4, TRPM5 and NFKB1, and a strong association has been established between the HumanMethylation 450 BeadChip and the GoldenGate Assay, of which the former is a more recent evolution rooted in the latter's technology [10]. It has also been argued that regions spanning several CpGs tend to be differentially methylated as opposed to individual nucleotides therefore we would anticipate several proximate CpG's to be associated with specific genes [11].

\section{Quantifying biases that affect the methylome}

One factor known to influence DNA methylation is gender, and this has also been observed in the autosomes [12]. Including child's sex as the only covariate in our 0-18 weeks GWG model did not result in any notable differences, although the GIF decreased from $\lambda=1.074$ for the crude model without covariates, to $\lambda=1.059$. Several studies have indicated that cell types, especially those found in cord blood, may substantially influence the methylome as well [13, 14]. In our study we found that controlling for cell type proportions, using a method described by Houseman et al. [14], improved the 


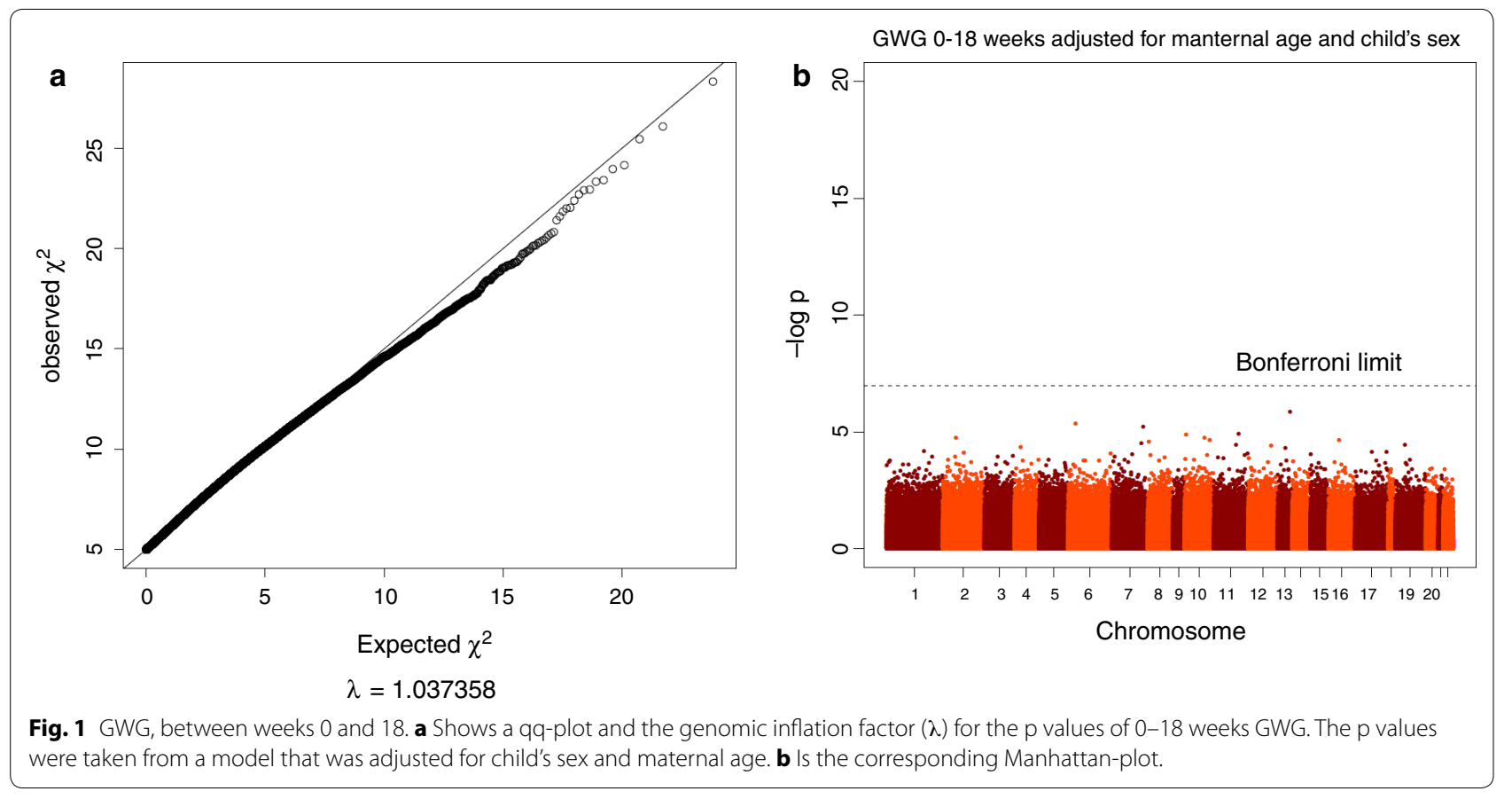

Table 2 Results for 0-18 weeks GWG adjusted for maternal age and child's sex

\begin{tabular}{|c|c|c|c|c|c|c|c|}
\hline Gene (\# CpG's) & GoldenGate CpG & Closest CpG & Coef & $\mathrm{Se}$ & GWG p value (adjusted) & GWG q value (450K) & GWG q value (GG) \\
\hline $\mathrm{MMP7}^{*}(7)$ & $\operatorname{cg} 10521988$ & cg04059146 & 0.00706 & 0.00466 & 0.1300039 (0.9100273) & 0.9198694 & 0.9244006 \\
\hline MMP7 (7) & cg20645973 & cg20645973 & -0.00141 & 0.005456 & $0.7955276(\sim 1.0)$ & 0.9600729 & 0.9720421 \\
\hline KCNK4 (26) & cg01352108 & cg01352108 & -0.00794 & 0.004879 & $0.1041649(\sim 1.0)$ & 0.9198694 & 0.9244006 \\
\hline KCNK4 (26) & cg25881850 & cg25881850 & -0.00386 & 0.004814 & $0.4233765(\sim 1.0)$ & 0.9373223 & 0.9244006 \\
\hline TRPM5 (46) & cg01581060 & cg01581060 & -0.00152 & 0.004361 & $0.7282958(\sim 1.0)$ & 0.9568195 & 0.9720421 \\
\hline TRPM5* (46) & cg03982381 & cg04691795 & -0.01572 & 0.00626 & $0.0122687(0.5643602)$ & 0.9198694 & 0.9244006 \\
\hline TRPM5* (46) & cg19265431 & cg10172068 & -0.02234 & 0.012938 & $0.012938(0.595148)$ & 0.9198694 & 0.9244006 \\
\hline NFKB1* (19) & cg06930505 & cg00790535 & -0.0176 & 0.007064 & $0.0129537(0.2461203)$ & 0.9198694 & 0.9244006 \\
\hline NFKB1* (19) & cg24413435 & cg12615753 & 0.00936 & 0.006383 & $0.1430112(\sim 1)$ & 0.9198694 & 0.9244006 \\
\hline
\end{tabular}

The table shows coefficient estimates (Coef), standard errors (Se) and p/q value estimations for the CpG loci linked to the genes found significantly differentially methylated in the ALSPAC study. The $p$ values are "raw" in the sense that they are unadjusted for multiple testing; $p$ values Bonferroni-adjusted for multiple testing according to the number of CpG's on the Illumina Humanmethylation450 platform associated with each respective gene is included in brackets. The q values were based on multiple test corrections performed for both the HumanMethylation450 platform [GWG q value (450K), 473,731 tests] and the Golden Gate platform [GWG q value (GG) 1,505 tests]. CpGs marked with * were not directly observed but flanking markers are reported which were associated within the gene body (all *-marked CpG's linked to the NFKB1 and TRPM5 genes) and promoter regions (*-marked CpG linked to the MMP7 gene).

model marginally with respect to GIF $(\lambda=0.998)$ compared with a crude model $(\lambda=1.074)$. However, including cell-type proportions did not lead to any differences with respect to significant $\mathrm{CpG}$ loci in any of the models tested, including the full model discussed above of which the GIF increased from $\lambda=1.042$ to $\lambda=1.206$. Another issue that may bias or influence results is the qualitycontrol performed on the methylome dataset. It has been shown that different filtering procedures could influence $p$ values if the signal is weak in potential findings [15].

\section{Strengths and weaknesses of the present study}

Our study strengths include a larger number of newborns than the ALSPAC study and genome-wide coverage of CpG loci. It can however not be concluded that there are no effects of GWG on the methylome since the total methylome in the human genome is assumed to consist of approximately 28 million loci [16], as compared to the 485,512 loci mapped by the HumanMethylation 450 platform. Moreover, the mean weight gain approximately 18 weeks after conception ( $\mathrm{kg} /$ week) for the 
mothers in our study $(0.16 \pm 0.17$, mean $\pm S D)$ was considerably less than for the mothers in the ALSPAC study $(0.32 \pm 0.17$, mean $\pm \mathrm{SD})$. Statistical power was also limited for small effect sizes; based on a post hoc power calculation assuming a regression model with $0-18$ week GWG, child's sex, maternal age and cell-type correction (9 explanatory variables in total) we achieved a power of $60 \%$ for median effect sizes $\left(R^{2}=0.0610\right)$. Small effects sizes (lower quartile, $R^{2}=0.0124$ ) resulted in a power below $1 \%$ ( $R^{2}$ refers to the proportion of variance of the corresponding methylation site explained by the variance of the regression model).

\section{Methods}

\section{Study population}

The Norwegian Mother and Child Cohort Study (MoBa) is a prospective population-based pregnancy cohort study conducted by the Norwegian Institute of Public Health [17, 18]. MoBa recruited pregnant women between 1999 and 2008, at approximately 18 weeks after conception and mothers could participate with more than one pregnancy, resulting in 95,200 mothers and 114,500 children in total. The participation rate of invited pregnant women was $40.6 \%$. Umbilical cord blood samples were collected at delivery and sent by post to the biobank at the Norwegian Institute of Public Health where DNA was extracted and stored at $-20^{\circ} \mathrm{C}$ until analysis [19]. The dataset used in the present study is a subset of the one described by Joubert et al. [1]. From that dataset a sub-sample was extracted consisting of a random sample of children (729 in total), included in the analyses by Joubert et al., but children sampled because they had asthma at 3 years were excluded.

The MoBa cohort was linked to the Medical Birth Registry of Norway for information on sex of the child and maternal age. We used information from questionnaires completed by MoBa participants around week 18 of their pregnancies to calculate maternal pre-pregnancy weight $(\mathrm{kg})$, and GWG ( $\mathrm{kg} /$ week) based on the difference between the mother's self-reported pre-pregnancy weight and current weight at 18 gestational weeks.

The MoBa study has been approved by the Regional Committee for Medical and Health Research Ethics, the Norwegian Data Inspectorate and the Institution Review Board of the National Institute of Environment Health Sciences, USA. Written informed consent was provided by all participants.

\section{DNA methylation technology}

Cord blood DNA methylation was measured using the Illumina Infinium HumanMethylation450 BeadChip (450K) (http://www.illumina.com). This assay was designed to conduct epigenome-wide association studies (EWAS), and includes 485,577 methylation (CpG) loci per sample at single-nucleotide resolution. This chip covers $99 \%$ of RefSeq genes, an average of 17 CpG sites per gene region across the promoter, $5^{\prime} \mathrm{UTR}$, first exon, gene body, and $3^{\prime}$ UTR. Further, the chip covers $96 \%$ of CpG islands, with additional coverage of island shores. Details regarding quality control can be found in the study by Joubert et al. [1] and is also outlined in Additional file 3.

\section{Statistical analysis}

The statistical analyses were performed using standard ordinary least squares regression on each autosomal CpG locus, effectually resulting in 473,731 regression models with $0-18$ weeks GWG as the explanatory variable.

The genomic inflation factor (GIF) $\lambda[6]$ for these models was calculated using the "regression"-based method (default) in the GenABEL package [20].

We used Bonferroni correction and q values to correct for multiple testing [9]. Covariates included in the adjusted model were child's sex and maternal age (continuous). In addition, we estimated cell type proportions (i.e. CD4, CD8, Gran, NK, Bcell, Mono) using a method suggested by Houseman et al. [14] as implemented in the minfi package [21]. These estimates were then added to the regression model described above as separate covariates. Several other covariates were also tested, and Additional file 1 contains qq-/Manhattan plots of the $\mathrm{p}$ values from a model containing the covariates: child's sex, maternal age, maternal education, maternal daily smoking during pregnancy, caesarian section and parity, (see Table 1 for summary information regarding these covariates) however no differences were detected and therefore the simple model proposed by Morales et al. [5] was presented as the main model.

\section{Additional files}

Additional file 1: The file contains a regression model diagnostics plot
including a qq-plot, distribution plot and a manhattan plot of p-values
resulting from a regression model based on 0-18 week GWG with covari-
ates child's sex, maternal daily smoking, maternal education, caesarian
section, parity and maternal age.

Additional file 2: A table in Microsoft Excel format containing information regarding the regression model based on 0-18 week GWG.

Additional file 3: Details regarding quality control of the methylation dataset.

\section{Authors' contributions}

JB, BKA, BRJ, MCM, CLP, SHE, SJL: wrote the manuscript. JB, BKA, MCM: initiated the project. JB, BKA, MCW, PM, SER: statistical analyses. BRJ, SHE, PM, CS, SJL, WN: acquisition of data. BRJ, MCW, SJL: quality control of methylation data. MCM, SHE, PM, CS, WN: collection of phenotypes. JB, BKA, MCW, SJL, WN: drafted and revised the manuscript. All authors read and approved the final manuscript. 


\section{Author details}

${ }^{1}$ Division of Epidemiology, Norwegian Institute of Public Health, Marcus Thranes gate 6, P.O. Box 4404, 0403 Oslo, Norway. ${ }^{2}$ Department of Molecular Biology, Institute of Clinical Medicine, University of Oslo, Oslo, Norway. ${ }^{3}$ National Institute of Environmental Health Sciences, MD A3-05, PO Box 12233, Research Triangle Park, NC 27709, USA. ${ }^{4}$ Public Health Sciences Division, Fred Hutchinson Cancer Research Center, Seattle, WA 98109, USA.

\section{Acknowledgements}

The Norwegian Mother and Child Cohort Study is supported by NIH (NIH/ NIEHS contract number N01-ES-75558, NIH/NINDS Grant No. 1 UO1 NS 047537-01 and Grant No. 2 UO1 NS 047537-06A1) and the Norwegian Research Council/FUGE (Grant Number 151918/S10). This study is supported in part by the Intramural Research Program of the NIH, NIEHS (ZIA ES049019), and in part by the Norwegian Research Council/Human Biobanks and Health (Grant Number 221097), in addition to the Norwegian Extra-Foundation for Health and Rehabilitation (Grant Number 2011.2.0218). We are grateful to all families participating in the Norwegian Mother and Child Cohort Study.

\section{Compliance with ethical guidelines}

\section{Competing interests}

The authors declare that they have no competing interests.

Received: 12 December 2014 Accepted: 22 July 2015

Published online: 29 July 2015

\section{References}

1. Joubert BR, Haberg SE, Nilsen RM, Wang X, Vollset SE, Murphy SK et al (2012) 450K epigenome-wide scan identifies differential DNA methylation in newborns related to maternal smoking during pregnancy. Environ Health Perspect 120(10):1425-1431

2. Engel SM, Joubert BR, Wu MC, Olshan AF, Haberg SE, Ueland PM et al (2014) Neonatal genome-wide methylation patterns in relation to birth weight in the Norwegian Mother and Child Cohort. Am J Epidemiol 179(7):834-842

3. Monte S, Valenti O, Giorgio E, Renda E, Hyseni E, Faraci M et al (2011) Maternal weight gain during pregnancy and neonatal birth weight: a review of the literature. J Prenat Med 5(2):27-30

4. Ludwig DS, Currie J (2010) The association between pregnancy weight gain and birthweight: a within-family comparison. Lancet 376(9745):984-990

5. Morales E, Groom A, Lawlor DA, Relton CL (2014) DNA methylation signatures in cord blood associated with maternal gestational weight gain: results from the ALSPAC cohort. BMC Res Notes 7:278

6. Yang J, Weedon MN, Purcell S, Lettre G, Estrada K, Willer CJ et al (2011) Genomic inflation factors under polygenic inheritance. Eur J Hum Genet EJHG 19(7):807-812
7. Listgarten J, Kadie C, Schadt EE, Heckerman D (2010) Correction for hidden confounders in the genetic analysis of gene expression. Proc Natl Acad Sci USA 107(38):16465-16470

8. Chen W, Gao G, Nerella S, Hultman CM, Magnusson PK, Sullivan PF et al (2013) MethyIPCA: a toolkit to control for confounders in methylomewide association studies. BMC Bioinform 14:74

9. Storey JD, Tibshirani R (2003) Statistical significance for genomewide studies. Proc Natl Acad Sci USA 100(16):9440-9445

10. Sandoval J, Heyn H, Moran S, Serra-Musach J, Pujana MA, Bibikova M et al (2011) Validation of a DNA methylation microarray for 450,000 CpG sites in the human genome. Epigenetics Off J DNA Methylation Soc 6(6):692-702

11. Lister R, Pelizzola M, Dowen RH, Hawkins RD, Hon G, Tonti-Filippini J et al (2009) Human DNA methylomes at base resolution show widespread epigenomic differences. Nature 462(7271):315-322

12. Liu J, Morgan M, Hutchison K, Calhoun VD (2010) A study of the influence of sex on genome wide methylation. PLoS One 5(4):e10028

13. Jaffe $A E$, Irizarry RA (2014) Accounting for cellular heterogeneity is critical in epigenome-wide association studies. Genome Biol 15(2):R31

14. Houseman EA, Accomando WP, Koestler DC, Christensen BC, Marsit CJ, Nelson $\mathrm{HH}$ et al (2012) DNA methylation arrays as surrogate measures of cell mixture distribution. BMC Bioinform 13:86

15. Wu MC, Joubert BR, Kuan PF, Haberg SE, Nystad W, Peddada SD et al (2014) A systematic assessment of normalization approaches for the Infinium 450K methylation platform. Epigenetics Off J DNA Methylation Soc 9(2):318-329

16. Stevens M, Cheng JB, Li D, Xie M, Hong C, Maire CL et al (2013) Estimating absolute methylation levels at single-CpG resolution from methylation enrichment and restriction enzyme sequencing methods. Genome Res 23(9):1541-1553

17. Magnus P, Irgens LM, Haug K, Nystad W, Skjaerven R, Stoltenberg C (2006) MoBa Study G: Cohort profile: the Norwegian Mother and Child Cohort Study (MoBa). Int J Epidemiol 35(5):1146-1150

18. Nilsen RM, Vollset SE, Gjessing HK, Skjaerven R, Melve KK, Schreuder P et al (2009) Self-selection and bias in a large prospective pregnancy cohort in Norway. Paediatr Perinat Epidemiol 23(6):597-608

19. Ronningen KS, Paltiel L, Meltzer HM, Nordhagen R, Lie KK, Hovengen R et al (2006) The biobank of the Norwegian Mother and Child Cohort Study: a resource for the next 100 years. Eur J Epidemiol 21(8):619-625

20. Aulchenko YS, Ripke S, Isaacs A, van Duijn CM (2007) GenABEL: an $\mathrm{R}$ library for genome-wide association analysis. Bioinformatics 23(10):1294-1296

21. Aryee MJ, Jaffe AE, Corrada-Bravo H, Ladd-Acosta C, Feinberg AP, Hansen KD et al. (2014) Minfi: a flexible and comprehensive Bioconductor package for the analysis of Infinium DNA methylation microarrays. Bioinformatics 30(10):1363-1369

\section{Submit your next manuscript to BioMed Central and take full advantage of:}

- Convenient online submission

- Thorough peer review

- No space constraints or color figure charges

- Immediate publication on acceptance

- Inclusion in PubMed, CAS, Scopus and Google Scholar

- Research which is freely available for redistribution 